\title{
Estudo de fatores associados à adesão ao tratamento não farmacológico em portadores de diabetes mellitus assistidos pelo Programa Saúde da Família, Ventosa, Belo Horizonte
}

\author{
A study of factors associated to non-pharmacological treatment \\ delivered by the Family Health Program in Ventosa, \\ Belo Horizonte, to carriers of diabetes mellitus
}

Thaís Silva Assunção ${ }^{1}$

Príscila Guedes Santana U rsine ${ }^{1}$

${ }^{1}$ Departamento de Fisioterapia, Centro Universitário de Belo Horizonte. Av. Prof. Mário Werneck 1685, Estoril. 30455-610 Belo Horizonte M G. bdthais@yahoo.com.br
Abstract Diabetes M ellitus is turning into one of the main public health problems in Brazil. Many factors may contribute to the low adherence to treatment. The objective of this study was to investigate the association between educational and social-economic factors and factors related to the health, the perception of disease, social support and adherence to non-pharmacological treatment of carriers of diabetes mellitus assisted in the family health program of the Health Center in Ventosa, Belo H orizonte. Data were collected in interviews with 164 diabetics who were waiting for assistance in the health center. Univariate analysis showed significant association between adhe sion to the non-pharmacological treatment and low income; place of residence; knowledge about consequences; motivation with regard to the treatment; making part of a diabetics group and orientation received from the nurse and the physiotherapist. M ultivariate analysis showed the variables " treatment motivation" and "part of some diabetics group", to be statistically significant as refers to the total adhesion $(p<0,05)$. The variables "knowledgeabout consequences" and "place of residence- high risk area" were statistically significant as refers to the total adhesion $(p<0,06)$, however presenting an inverse relation.

Key words Diabetes mellitus, Treatment adhesion, Family Health Program
Resumo 0 diabetes mellitus vem setornando um dos principais problemas de saúde pública no Brasil. Há vários fatores que podem contribuir para a baixa adesão ao tratamento. 0 objetivo desteestudo foi investigar a associação entre fatores educacionais, demográficos, socioeconômicos, de saúde, percepção da doença, suporte social e adesão ao tratamento não farmacológico em portadores de diabetes mellitus assistidos pelo Programa Saúde da Família do Centro de Saúde Ventosa, em Belo H orizonte. As informações foram obtidas através de entrevistas com 164 diabéticos que estavam aguardando 0 acolhimento do Centro de Saúde. $\mathrm{Na}$ análise univariada, verificou-se associação significante entre a adesão ao tratamento não farmacológico e baixa renda, local de residência, conhecimento sobre complicações, motivação com o tratamento, fazer parte de grupo de diabéticos e orientações recebidas pelo enfermeiro e fisioterapeuta. A pós análise multivariada, as variáveis "motivação com o tratamento" e "faz parte de algum grupo de diabético" foram estatisticamente significativas para a adesão total $(p<0,05)$. As variáveis "conhecimento sobre as complicações" e "local de residência - área de elevado risco" obtiveram limiar de significância estatística em relação à adesão total $(p=0,06)$, apresentando, no entanto, relação inversa.

Palavras-chave Diabetes mellitus, Adesão ao tratamento, Programa Saúde da Família 
Introdução

O Brasil assiste, desde a década de 1960, a sua transição demográfica eepidemiológica caracterizada pelo envelhecimento populacional eaumento das doenças crônico-degenerativas ${ }^{1}$. Dentre as doenças crônicas mais freqüentes, destaca-se 0 diabetes mellitus (DM ), que consiste em uma disfunção metabólica crônica, grave, deevolução lenta e progressiva, caracterizada pela falta ou produção diminuída de insulina e/ou da incapacidade dessa em exercer adequadamente seus efeitos metabólicos, levando à hiperglicemia e glicosúria².

Um estudo multicêntrico desenvolvido na população brasileira encontrou uma prevalência geral de DM de $7,6 \%$ em pessoas de 30 a 69 anos $^{3}$. A alta prevalência associada às complicações crônicas inerentes à doença torna o DM um dos principais problemas de saúde pública em nosso país, representando alto custo social e grande impacto na morbi-mortalidade da população 4 .

$\mathrm{N}$ a rede pública de saúde, a porta de entrada para o portador de DM é a Unidade Básica de Saúde, que está estruturada de acordo com 0 Programa Saúde da Família (PSF) 5 . O PSF éuma estratégia que tem como principal propósito re organizar a prática da atenção básica à saúde e substituir o modelo tradicional de assistência, priorizando ações de prevenção, promoção erecuperação da saúde, de forma integral e contínua. 0 atendimento é prestado na unidade básica de saúde ou no domicílio pelos profissionais da saúde que compõem as Equipes de Saúde da Família. Esses profissionais ea população acompanhada criam vínculos de co-responsabilidade, o que facilita a identificação dos problemas de saúde da comunidade e seu atendimento ${ }^{5}$.

De acordo com a Sociedade Brasileira de Diabetes, o tratamento básico e o controle da doença consistem, primordialmente, na utilização de uma dieta específica baseada na restrição de alimentos ricos em carboidratos, gorduras e proteínas, atividadefísica regular eno uso adequado de medicação $0^{6}$. Entretanto, a adesão a esse tratamento exige comportamentos de alguma complexidade que devem ser integrados na rotina diária do portador de DM 7 .

A adesão ao tratamento em pacientes crônicos representa a extensão no qual o comportamento da pessoa coincide com o aconselhamento dado pelo profissional de saúde, contemplando três estágios: 1) concordância, no qual o indivíduo, inicialmente, concorda com o tratamento, seguindo as recomendações dadas pelos profissionais da saúde. Existe, freqüentemente, uma boa supervisão, assim como uma elevada eficácia do tratamento; 2) adesão, fase de transição entre os cuidados prestados pelos profissionais de saúde e o autocuidado, no qual, com uma vigilância limitada, o doente continua com o seu tratamento, o que implica uma grande participação e controle da sua parte; 3) manutenção, quando, já sem vigilância (ou vigilância limitada), o doente incorpora o tratamento no seu estilo de vida, possuindo um determinado nível de autocontrole sobre os novos comportamentos ${ }^{8}$.

A cronicidade da doença, associada às características do regime terapêutico e às responsabilidades do doente, pode contribuir para a baixa adesão vulgarmente encontrada nos diabéticos ${ }^{7}$. Segundo estudo desenvolvido por Assunção et al. ${ }^{9}$, apenas $25 \%$ dos pacientes diabéticos que receberam orientações quanto a prática de atividade física realizaram algum tipo de atividade; e dos pacientes que receberam orientações quanto à dieta, apenas metade seguiu as recomen dações. Outro estudo realizado por Assunção et al. ${ }^{4}$ verificou que cerca de $53 \%$ dos pacientes relataram ter feito dieta nos últimos quinze dias, $10 \%$ não estavam fazendo nenhum tipo de tratamento e $26 \%$ afirmaram usar apenas medicamento no tratamento da doença.

0 presente estudo teve como objetivo verificar sefatores demográficos, socioeconômicos, de saúde, percepção da doença e suporte social estão associados à adesão ao tratamento não farmacológico em portadores de DM assistidos pelo PSF-Ventosa, o que poderá auxiliar no planejamento de estratégias de prevenção e intervenção mais adequadas para abranger essa população e suas peculiaridades.

\section{M ateriais e métodos}

\section{Local do estudo}

Este estudo foi realizado na área de abrangência do Centro de Saúde Ventosa (CSV), situado no bairro Jardim América, Distrito Sanitário Oeste, em Belo Horizonte (MG), cidade de grande porte e que possui atualmente 2.238.526 habitantes.

A Secretaria Municipal deSaúde(SM SA-BH), órgão da administração direta da Prefeitura M unicipal de Belo Horizonte, optou pela reorganização dos serviços de saúde em base territorial através da definição de nove distritos sanitários (Venda Nova, Barreiro, Leste, Nordeste, Oeste, Pampulha, Centro-sul, N oroeste e N orte). Cada 
um dos nove distritos sanitários, que correspondem às Administrações Regionais da Prefeitura deBelo H orizonte, tem definido certo espaço geográfico, populacional e administrativo de sua abrangência.

\section{Amostra}

A população do estudo foi composta por 164 diabéticos assistidos pelo PSF do Centro de Saúde Ventosa. Para o cálculo do tamanho da amostra, usamos a seguinte fórmula ${ }^{10}$ :

$$
n=\frac{N}{\left(\frac{E}{Z \sigma}\right)^{2}(N-1)+1}
$$

Fixando:

$N=1663$ (tamanho da população de diabéticos cadastrados ao PSF/Ventosa);

$\mathrm{E}=0,1453$ (margem de erro)

$\sigma=1,00$ (desvio padrão máximo que podemos ter para uma amplitude de 0 a 2);

$Z=1,96$ (valor tabelado da distribuição normal para 95\% de confiança)

Temos que $n=164$

As entrevistas foram realizadas no Centro de Saúde Ventosa com os indivíduos que estavam na fila de espera para o acolhimento, sendo incluídos no estudo indivíduos portadores de DM tipo 1 e2, capazes de se comunicar verbalmente, com idade acima de 18 anos e que consentiram em participar da pesquisa.

\section{Coleta de dados}

A coleta de dados foi realizada por três alunos do nono período do curso de Fisioterapia do Centro Universitário de Belo Horizonte, os quais foram devidamente treinados para a utilização dos instrumentos propostos. Para tanto, foi realizada uma discussão teórica sobre o tema de estudo, aplicabilidade de cada item do questionário e estratégia para a coleta de dados. Foram realizadas também simulações de situações de campo, nas quais os pesqui sadores aplicaram os instrumentos entre si e, em seguida, em seis pacientes da Clínica-Escola do Uni-BH, portadores de DM, para avaliação crítica da compreensão, ordem das questões, identificação de dúvidas a respeito de preenchimento e, posteriormente, cálculo da confiabilidade inter-examinador. Para cálculo da confiabilidade inter-exami- nador, foi utilizada a estatística Kappa, sendo a confiabilidade classificada como quase perfeita (Kappa=0,96)

O questionário semi-estruturado desenvolvido para este estudo tinha como objetivo obter informações referentes às características demográficas (sexo, idade elocal da residência), socioeconômicas (classe social e renda mensal), de saúde (tipo de diabetes, o tempo de diagnóstico da doença, uso de medicamento e controle de glicose), percepção da doença (nível de conhecimento e aceitação da doença, motivação com 0 tratamento), suporte social (apoio da família, orientações com relação ao diabetes, fazer parte de algum grupo de diabéticos no PSF/ Ventosa) e adesão ao tratamento proposto para o DM.

Quanto às características socioeconômicas, a renda mensal familiar foi registrada como os somatórios das rendas individuais de todas as pessoas economicamente ativas. A classe social foi categorizada em A, B, C, D eE, deacordo com a classificação da ABIPEM E (Associação Brasileira de Institutos de Pesquisa de M ercado) ${ }^{11}$.

O local de residência foi categorizado como elevado, médio e baixo risco com os critérios do Índice de Vulnerabilidade Social (IVS) utilizado pela SM SA/PBH . O IVS é uma combinação de diferentes variáveis num indicador quebusca resumir informações relevantes que traduzem as desigualdades intra-urbanas, apontando áreas prioritárias para intervenção e alocação de recursos, o que favorece a proposição de ações intersetoriais.

Quanto às questões referentes à percepção da doença, o nível de conhecimento foi classificado como nenhum, básico (conhecimento de apenas uma forma de tratamento - dieta ou atividade física ou medicação), moderado (conhecimento de duas formas de tratamento), ou avançado (conhecimento dos três formas de tratamento).

A adesão ao tratamento não farmacológico para o DM foi definida por resposta positiva em ambas as perguntas relacionadas à realização de atividadefísica regular econtrolealimentar. A adesão parcial foi definida por resposta positiva em uma das perguntas (atividadefísica regular ou controle alimentar) e nenhuma adesão foi definida por resposta negativa em ambas as perguntas.

A aplicação do questionário foi feita no Centro de Saúde Ventosa, onde os indivíduos eram abordados e convidados a participarem do estudo. A pós a assinatura do termo de consentimento livree esclarecido pelos participantes, iniciouse a aplicação do questionário em local reservado sem a presença defamiliares, curiosos ou trân- 
sito de pessoas para que o mesmo não se dispersasse ou ficasse constrangido ao responder as perguntas e omitisse algum dado.

\section{Variáveis do estudo}

A variável dependente deste trabalho foi à adesão ao tratamento não farmacológico, definida por resposta positiva em ambas as perguntas: "Faz al gum tipo deatividadefísica regular?" e "Faz algum tipo de controle alimentar?". Foi investigada a associação entre a adesão ao tratamento não farmacológico e cinco conjuntos de variáveisindependentes: características socioeconômicas (renda mensal e classe social), demográficas (sexo, idade e local da residência - área de elevado, médio, baixo risco), referentes à saúde (tipo de diabetes, o tempo de diagnóstico da doença, uso demedicamento econtrole deglicose), referentes à percepção da doença (nível de conhecimento eaceitação da doença e suas complicações, motivação do paciente com o tratamento) e referentes ao suporte social (receber apoio da família no controle do $D M$, receber orientações com relação à doença, fazer parte de algum grupo para diabéticos no PSF/Ventosa).

\section{Análise de dados}

Foi elaborado um banco de dados utilizando o programa computacional M icrosoft Excel. A pós a tabulação dos dados, esses foram exportados para o software M initab for W indows versão 14. Através desse software, foram realizadas as estatísticas descritivas das variáveis independentes e variável dependente.

Em seguida, foi realizada a análise univariada através do teste não paramétrico de Kruskal Wallis. As variáveis estatisticamentesignificantes $(p<0,20)$ na análise univariada ebiologicamente plausíveis foram examinadas simultaneamente usando-se a regressão logística nominal para identificação dos fatores associados à adesão ao tratamento não farmacológico dessa população.

\section{Resultados}

Durante o período proposto, 164 indivíduos foram entrevistados ( $71,9 \%$ do sexo feminino e $28 \%$ do sexo masculino). A maioria tinha idade entre 60-75 anos (31,7\%), com média de 57,7 anos e desvio padrão de 12,9 anos.

Quanto à porcentagem de adesão ao tratamento não farmacológico, a maioria dos indiví- duos entrevistados adere totalmente (52,44\%), $38,41 \%$ aderem parcial mentee $9,15 \%$ não aderem.

A distribuição das características socioeconômicas, demográficas e fatores relativos à saúde associados à adesão ao tratamento não farmacológico dos portadores de DM está apresentada na Tabela 1. Verificou-se associação significante $(p<$ 0,20 ) entre a adesão ao tratamento não farmacológico e baixa renda (até um salário), local deresidência (área de elevado risco), controle eventual de glicose e conhecimento sobre as complicações do DM relacionadas à visão. As outras variáveis não apresentaram significância estatística.

A Tabela 2 representa a distribuição dos fatores relativos à avaliação subjetiva de saúde e ao suporte social. As variáveis "motivação com 0 tratamento", "fazer parte de al gum grupo de diabéticos" e"orientações recebidas pelo enfermeiro e fisioterapeuta" apresentaram associação significante com a adesão ao tratamento não farmacológico $(p<0,20)$. As outras variáveis não apresentaram significância estatística.

Os resultados finais da análise de regressão nominal das variáveis estatisticamentesignificantes na análise univariada encontram-se nas tabelas 3 , 4 e 5 . 0 s fatores que apresentaram asso-

Tabela 1. Distribuição das características socioeconômicas, demográficas e fatores relativos à saúde associados à adesão ao tratamento não farmacológico dos portadores de diabetes mellitus. Belo Horizonte, M inas Gerais, Brasil, 2006.

\begin{tabular}{lcl}
\hline \multicolumn{1}{c}{ Variáveis } & $\mathrm{H}$ & $\mathrm{P}$ \\
\hline Sexo & 0,10 & 0,746 \\
Idade & 0,76 & 0,683 \\
Renda mensal (até um salário) & 3,42 & $0,181 *$ \\
Local de residência (elevado risco) & 4,50 & $0,105 *$ \\
Classe social & 3,02 & 0,221 \\
Controle eventual da glicose & 9,95 & 0,019 \\
Tipo de diabetes & 0,62 & 0,732 \\
Tempo de diagnóstico & 2,11 & 0,550 \\
Toma medicamento & 1,56 & 0,212 \\
Se sim, qual & 1,56 & 0,212 \\
Você sabe das complicações: & & \\
$\quad$ Sim & 0,36 & 0,547 \\
Amputação & 0,43 & 0,512 \\
Visão & 2,01 & $0,156 *$ \\
\hline
\end{tabular}




ciação com a adesão ao tratamento não farmacológico, após análise multivariada, foram: motivação com o tratamento; fazer parte de algum grupo de diabéticos; conhecimento sobreas complicações relacionadas à amputação e problemas na visão elocal residência - área de elevado risco.

A variável "motivação com o tratamento" obteve significância estatística $(p<0,05)$ em relação à adesão parcial, como mostra a Tabela 3. A resposta dada pelo indivíduo com motivação está diretamente relacionada à adesão parcial ( 0 dds $>1$ ). Pela Odds, podemos dizer que um indivíduo que se diz motivado tem uma chance 6,60 vezes maior de ser aderente ao tratamento parcial do que o indivíduo que não adere.

Apenas a variável "faz parte de algum grupo de diabéticos" foi estatisticamente significativa para a adesão total $(p<0,05)$, como mostra a Tabela 4 .

A participação em algum grupo de diabéticos está relacionada diretamente com a adesão total (Odds $>1$ ). Logo podemos dizer queum indivíduo quediz participar dealgum grupo tem uma chance 5,54 vezes maior de ser aderente ao tratamento total do que o indivíduo que adere parcialmente.

Asvariáveis"conhecimento sobreas complicações" e"motivação com o tratamento" obtiveram quasesignificância estatística ( $p<0,06)$. 0 conhecimento sobre complicações, caso o tratamento não seja bem feito, está inversamente relacionado com a adesão total ( 0 dds $<1)$. Pela Odds, podemos dizer queum indivíduo quediz conhecer al guns problemas acarretados pelo DM tem uma chance2,17 vezes menor deaderir ao tratamento total secomparado ao tratamento parcial $(1 / 0,46=2,17)$. A resposta dada pelo indivíduo em motivação está diretamenterelacionada à adesão total ( 0 dds $>1$ ) . Pela Odds, podemos dizer que um indivíduo que se diz motivado tem uma chance 3,06 vezes maior de aderir ao tratamento total que ao parcial.

A variável local deresidência- área deelevado risco apresentou-secomo quasesignificativa àadesão total $(p=0,96)$, como mostra a Tabela 5 . A relação é inversa e, portanto, um indivíduo que mora em área de elevado risco teria uma chance 1,85 vezes menor deaderir ao tratamento total do que outro que mora em uma área de menor risco. $(1 / 0,54=1,85)$

\section{Discussão}

A pesar das limitações previstas na utilização de um roteiro de entrevista estruturado, sujeito às
Tabela 2. Distribuição dos fatores relativos à percepção da doença e ao suporte social associados à adesão ao tratamento não farmacológico dos portadores de diabetes mellitus. Belo Horizonte, M inas Gerais, Brasil, 2006.

\begin{tabular}{|c|c|c|}
\hline Variáveis & $\mathrm{H}$ & $\mathrm{p}$ \\
\hline Você se sente motivado & 16,22 & $0,00 *$ \\
\hline Conhecimento sobre a doença & 2,24 & 0,326 \\
\hline $\begin{array}{l}\text { Você tem algum problema em } \\
\text { aceitar }\end{array}$ & 0,08 & 0,780 \\
\hline Faz parte de algum grupo & 16,46 & $0,000 *$ \\
\hline \multicolumn{3}{|c|}{ Orientações com relação a diabetes } \\
\hline M édico & 1,23 & 0,267 \\
\hline Enfermeiro & 1,73 & $0,188 *$ \\
\hline Fisioterapeuta & 3,73 & $0,053 *$ \\
\hline Relação com a família & 0,19 & 0,660 \\
\hline Família sabe sobre os cuidados & 0,00 & 0,947 \\
\hline $\begin{array}{l}\text { Você sente interesse de sua } \\
\text { família em ajudar }\end{array}$ & 0,03 & 0,874 \\
\hline
\end{tabular}

$\mathrm{H}=$ Kruskal Wallis; ${ }^{\mathrm{p}}<0,20$
Tabela 3. Resultado da análise multivariada em relação à não adesão e adesão parcial. Belo Horizonte, M inas Gerais, Brasil, 2006.

\begin{tabular}{lcc}
\hline Variável & $p$ & Odds Ratio \\
\hline M otivação para o tratamento & 0,003 & 6,60 \\
\hline$p<0,05$ & & \\
& & \\
\hline
\end{tabular}

Tabela 4. Resultado da análise multivariada em relação à adesão parcial e adesão total. Belo H orizonte, M inas Gerais, Brasil, 2006.

\begin{tabular}{lcc}
\hline \multicolumn{1}{c}{ Variáveis } & $\mathrm{p}$ & Odds Ratio \\
\hline M otivação com o tratamento & 0,067 & 3,06 \\
$\begin{array}{l}\text { Fazer parte de algum grupo } \\
\text { de diabéticos }\end{array}$ & 0,002 & 5,54 \\
$\begin{array}{l}\text { Conhecimento sobre as } \\
\text { complicaçóes }\end{array}$ & 0,063 & 2,17 \\
\hline $\mathrm{p}<0,05$ & &
\end{tabular}




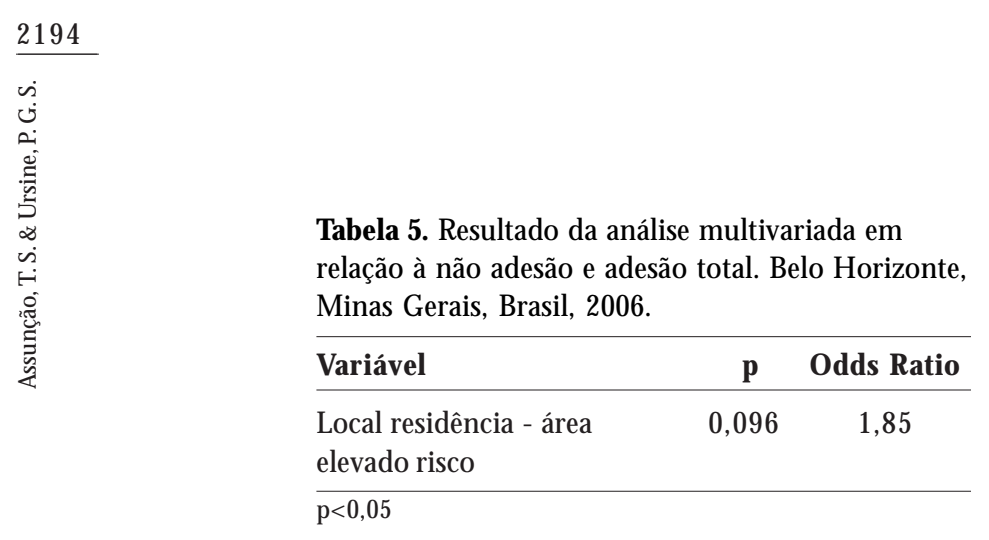

distorções etendenciosidades do informante, bem como a possíveis falhas inerentes ao procedimento decoleta dedados, verifica-sequeo instrumento utilizado ofereceu dados relevantese coerentescom aqueles encontrados na literatura da área.

Os resultados do presente estudo mostraram uma maior proporção de mulheres portadoras de DM , sendo quase três vezes maior quea de homens. Araújo et al. ${ }^{12}$ e Assunção et al. ${ }^{9}$, em estudostransversais com diabéticos atendidos em nível primário em Pelotas/ RS, também encontraram maior proporção de mulheres diabéticas (70,6\% e 76,1\%, respectivamente). Entretanto, em nosso país, em 1989, foi realizado um Estudo Multicêntrico, pelo M inistério da Saúde, em nove capitais brasileiras, indicando que a proporção foi semelhanteentre homens e mulheres, respectivamente 7,5 e $7,6 \%{ }^{3}$.

Por ser uma amostra de conveniência, os achados de nosso estudo podem sugerir uma maior preocupação das mulheres com sua própria saúde e acesso aos serviços. Isso porque, desde a perspectiva da divisão sexual do trabaIho, a tarefa de providenciar assistência médica à família é vista como atributo feminino, vindo a aumentar o contato da mulher com o serviço de saúdeegarantindo-Iheacesso facilitado. Goldenberg et al. ${ }^{13}$, avaliando o diabetes auto-referido no município deSão Paulo, verificaram, entre os pacientes diagnosticados, que as mulheres também freqüentaram mais os serviços de saúde.

A maior parte dos participantes encontravasenafaixa etária de 60 a 75 anos (31,7\%), o quese aproxima do observado em outros estudos ${ }^{9,14}$. No Brasil, observa-se um processo de transição demográfica e epidemiológica, levando a um aumento do número de idosos e também a um acréscimo significativo da expectativa de vida da população brasileira. Com o processo de enveIhecimento populacional, ocorreuma ruptura do mercado de trabalho, ocasionada pelo advento da aposentadoria. Com isso, o idoso tem um maior tempo livre e, conseqüentemente, uma maior disponibilidade de freqüentar os serviços de saúde, aumentando, assim, o acesso aos serviços médicos ${ }^{15}$.

Em relação à variável de desfecho, verificou-se que a maioria dos indivíduos entrevistados neste estudo aderiu totalmente ao tratamento não farmacológico (52,44\%). Embora atualmente sejam vários os estudos que se debruçam sobre a adesão, essa continua a ser um problema, estimando-se um nível aproximado de $50 \%$ de adesão às recomendações dos profissionais de saúde ${ }^{16}$.

A alta taxa de adesão encontrada no presente estudo pode ser explicada pelo fato da maioria dos entrevistados serem participantes de grupos de diabéticos e estarem motivados com o tratamento proposto, de acordo com análise multivariada.

A relação encontrada entre motivação e adesão ao tratamento em pacientes diabéticos está em acordo com outros estudos que afirmam que a motivação é uma das estratégias para melhorar a adesão ao tratamento em pacientes diabéticos juntamente com a prontidão para a modificação do estilo de vida, aceitação da doença e apoio familiar ${ }^{17}$. Santos et al. ${ }^{18}$ também preconizaram a necessidade da adoção de uma intervenção que aumente a motivação dos pacientes, familiares, médicos e das pessoas que convivem com os portadores de DM , requisitos fundamentais para um bom controle da doença.

Para tanto, é importante conhecer os fatores intrínsecos e extrínsecos que podem influenciar a motivação. No caso do DM, os fatores intrínsecos são a própria motivação do paciente no controle da doença. Dentre os fatores extrínsecos, podemos destacar a convivência com pessoas próximas, pois isso é motivo de extrema satisfação, fazendo com que os encontros freqüentes para a realização de al guma atividade tornem-se uma rotina em suas vidas, o que também Ihes proporcionará uma ajuda no controle metabólico. N este caso, 0 apoio e incentivo das pessoas próximas são indispensáveis ${ }^{19}$.

A participação em grupos operativos no PSF favorece o apoio social ao indivíduo portador de diabetes e a ação integrada da equipe multiprofissional em processos de educação em saúde ${ }^{16}$.

O apoio social é um processo recíproco, ou seja, gera efeitos positivos tanto para o sujeito que recebe como também para quem oferece 0 apoio. Esse apoio social pode contribuir parauma melhor adesão ao tratamento, uma vez que oferece aos integrantes do grupo ajuda emocional, realização de atividades conjuntas, interação so- 
cial, ajuda profissional, dos serviços de saúde e acesso a novos contatos, levando à interação com pessoas que até então não faziam parte da rede social do indivíduo. Os grupos ajudam não só no tratamento propriamente dito, mas também na parte psicológica, em que o paciente podecolocar seus problemas em debate, recebendo respostas e condutas corretas a serem utilizadas, além de ouvir outros indivíduos com problemas semelhantes ou mesmo opostos e tirar várias conclusões e experiências de vida, o que torna o tratamento mais dinâmico e completo ${ }^{16}$.

Entende-se por educação em saúde quaisquer combinações de experiências de aprendizagem delineadas com vistas a facilitar ações voluntárias conducentes à saúde ${ }^{20}$. 0 processo de educação em saúde consiste, basicamente, em cinco etapas: inicia-se com a escolha adequada de técnicas e estratégias para o processo de aprendizado; em seguida, ocorre a absorção da informação que pode ser resultante da internalização dos conhecimentos adquiridos ou simplesmente da memorização. A internalização acontece quando o indivíduo é capaz de interpretar a importância da informação recebida e relacioná-la com sua vida particular, dos familiares e ou da comunidade. Por fim, ocorre a aplicação dessas informações, ocorrendo a promoção da saúde, com conseqüente mudança no estilo de vida ${ }^{21}$.

Caso haja uma falha em algumas dessas etapas, o processo de educação em saúde fica prejudicado. Isso pode explicar a relação inversa encontrada em nosso estudo entre o conhecimento sobre complicações do DM e a adesão total. Os fatores que podem ter contribuído para tal achado podem ser o conhecimento do profissional de saúde a respeito da doença e a estratégia utilizada para passar a informação sobre o tratamento.

O profissional da saúde tem um papel importante no processo de adesão ao tratamento, já que atua como agente facilitador e mobilizador através da conscientização, mudança de comportamento e desenvolvimento da capacidade e habilidade do indivíduo para o autocuidado, adequando seus conhecimentos experiências à pratica clínica eà realidade do paciente ${ }^{22}$. Para isso, os profissionais de saúde necessitam de ter um conhecimento avançado sobre o controle, a prevenção e as complicações da doença, sendo responsáveis por propiciar condições favoráveis ao processo de aquisição de conhecimentos e possíveis mudanças comportamentais por parte do indivíduo diabético. Além disso, o entendimento pelo indivíduo da prática educativa e as interfaces estabelecidas entre esta e o profissional de saúde, favorecem o desenvolvimento de atitudes pessoais que se associam à mudança no estilo de vida ${ }^{22}$. No entanto, no nosso estudo não foram avaliados o conhecimento nem a forma como os profissionais da saúde passavam a informação a seus pacientes, sendo esse um dos fatores limitantes da pesquisa.

A informação é um fator-chave para que os pacientes possam participar ativamente nas decisões acerca da sua saúde ${ }^{23}$. Para tal, estes necessitam de trocar informações com o seu profissional de saúde, sobre as quais farão a respectiva integração, compreensão e avaliação. 0 fornecimento de informação é um aspecto considerado básico para que a pessoa tome decisões conscientes sobre a sua saúde, permitindo que esta enfrente as dificuldades e incertezas, e mantenha a sua máxima autonomia ${ }^{24}$.

Embora a adesão ao tratamento dependa da absorção da informação, o conhecimento ou informação sobre a doença não funciona por si só como um preditor da adesão. Um estudo desenvolvido por Chan \& M olassiotis ${ }^{25}$ analisou a relação entreo conhecimento sobre diabetes eadesão e demonstrou a existência de uma grande distância entre aquilo queéensinado aos pacientes a fazer e aquilo que eles realmente fazem. A maioria dos participantes apresentou boas notas no conhecimento da doença, mas perderam pontos, quando se trata de aplicar esse conhecimento nos seus hábitos diários. Esses resultados sugerem que o conhecimento não é garantiaúnica para a mudança de comportamentos nos doentes diabéticos.

Em relação à condição socioeconômica, este estudo demonstrou que os pacientes portadores de DM residentes em área de alto risco tiveram menor adesão ao tratamento não farmacológico, 0 que está em acordo com a literatura ${ }^{26-28}$.

Pace et al ${ }^{26}$ demonstraram ser a dieta e o fator financeiro as maiores dificuldades encontradas pelos pacientes no tratamento do DM. N esse estudo, verificou-se que existe uma relação entre as duas dificuldades, visto que uma melhor renda poderia facilitar a aquisi ção de alimentos.

Para que a adesão do diabético ao programa alimentar proposto seja satisfatória, éimportante considerar seu estilo de vida, rotina de trabal ho, nível socioeconômico, tipo de diabetes, medicação prescrita e hábitos alimentares anteriores ao DM . A adaptação da dieta aos hábitos alimentares preexistentes, sempre que possível, é a meIhor conduta, pois, além do indivíduo, a família ficará mais integrada ao tratamento. Do ponto de vista econômico, o uso de alimentos já roti- 
neiros e que sejam adequados oferece maior flexibilidadena escolha eimpede a substituição por outros, às vezes impróprios e mais caros ${ }^{27}$.

Em relação à atividadefísica, o estudo deSilva \& Lima ${ }^{28}$ mostrou que $78,7 \%$ dos participantes que aderiram à atividade física foram consideradosem uma condição socioeconômica muito boa. Monteiro et al. ${ }^{19}$ preconizaram também que 0 comportamento sedentário aumenta com a idadeeémaior entreaquelescom menor poder aquisitivo, o que corrobora os nossos resultados.

Uma corrente médica bem antiga defende a utilização de exercícios físicos como forma de prevenção, controle e procedimento terapêutico empregado em inúmeras doenças humanas. N esse contexto, énecessário considerar o cotidiano das pessoas, uma vez que as poucas opções de lazer determinadas por condições socioeconômicas precárias e as estruturas familiares voltadas unicamente para a própria subsistência são aspectos que limitam a relação entre o indivíduo, o exercício físico ea saúde ${ }^{19}$.

\section{Conclusão}

Com base nos resultados obtidos com esta investigação, pôde-se concluir que há uma grande adesão ao tratamento não-farmacológico no portador de DM que procura auxílio do Centro de Saúde Ventosa.

Os fatores que apresentaram associação com a adesão ao tratamento não-farmacológico foram: motivação com o tratamento, fazer parte dealgum grupo de diabéticos, conhecimento sobreas complicações do diabetes e morar em local de elevado risco.

Entende-se que há, ainda, a necessidade de mais estudos queavaliem os possíveis fatores que podem influenciar na adesão ao tratamento nãofarmacológico no DM .

\section{Colaboradores}

TS Assunção trabalhou na pesquisa, metodologia e redação do artigo e PGS Ursine trabalhou na concepção e redação final do artigo. 


\section{Referências}

1. Lopes FAM, Oliveira FA. Fatores de risco para o desenvolvimento do pé diabético em sujeitos atendidos pelo programa saúde da família (PSF). Patge 2004; 9(15):154-166.

2. Maia FFR, Araújo LR. Projeto "Diabetes Weekend" Proposta de educação em diabetes mellitus tipo 1. Arq Bras Endocrinol M etab 2002; 46(5):550-556.

3. Malerbi DA, Franco LJ. Multicenter study of the prevalence of diabetes mellitus and impaired glucose tolerance in the urban Brazilian population aged 30-69 years. Diabetes Care 1992; 15 (11):1509-1516.

4. Assunção MCF, Santos IS, Gigante DP. Atenção primária em diabetes no Sul do Brasil: Estrutura, processo e resultado. Rev. Saúde Pública 2001; 35(1):88-95.

5. Brasil. Ministério da Saúde. Programa Saúde da Família. Informe Técnico 1993. [acessado 2005 abr 13]. Disponível em: http://www.saude.gov.br

6. Brasil M inistério da Saúde. Manual de hipertensão arterial e diabetes mellitus. Informe Técnico 2002. [acessado 2005 jun 29]. Disponível em: http:// www.diabetes.org.br

7. Cox DJ, Gonder FL. Major Developments in Behavioral Diabetes Research. Journal of Consulting and Clinical Psychology 1992; 60(4):628-638.

8. Soares MC, Ribeiro A, Lima P, Ribeiro J. 0 efeito do fornecimento da informação na ansiedade pós-operatória numa população portuguesa de pacientes candidatos a cirurgia cardíaca. In: Actas do 20 congresso Nacional de Psicologia da Saúde; 1997; Lisboa.

9. Assunção MCF, Santos IS, Costa JS. Avaliação do processo da atenção médica: adequação de pacientes com diabetes mellitus. Cad Saúde Pública 2002; 18(1):205-211.

10. M orettin LG. Inferência. Estatística básica. São PauIo: Makron Books; 2000.

11. 11. Associação Brasileira de Institutos de Pesquisa de M ercado. [acessado 2005 set 05]. Disponível em: http://www.abep.org

12. Araújo RB, Santos I, Cavaleti MA, Costa JSD, Béria JU. Avaliação do cuidado prestado a pacientes diabéticos em nível primário. Rev. Saúde Pública 1999; 33(2):24-32.

13. Goldenberg P, Franco LJ, Pagliaro H, Silva RS, Santos CA. Diabetes mellitus auto-referido no município de São Paulo: prevalência e desigualdade. Cad Saúde Pública 1996; 12(1):37-45.

14. Khawali C, Andriolo A, Ferreira SRG. Benefícios da atividade física no perfil lipídico de pacientes com diabetes tipo 1.Arq Bras Endocrinol M etab 2003; 47(1):49-54.

15. Patarra NL. Mudanças na dinâmica demográfica. In: Monteiro CA. Velhos e novos males da saúde no Brasil: a evolução do país e de suas doenças. São PauIo: Hucitec; 1995. p.67-80.
16. Braga MRM GCS. Estudo dos conhecimentos e representações de doença associados à adesão terapêutica nos diabéticos tipo 2 [dissertação]. São Paulo (SP): Instituto de Educação e Psicologia,U niversidade do Minho; 2003.

17. Koenigsberg MR, Bartle TD, Cramer S. Facilitating treatment adherence with lifestyle changes in diabetes. American Family Physician 2004; 69(2):309-324.

18. Santos ECB, Zanetti ML, Otero LM, Santos MA. O cuidado sob a ótica do paciente diabético e de seu principal cuidador. Rev. Latino- americana Enfermagem 2005; 13(3):397-406.

19. Monteiro LH, Gonçalves A, Padovani RC, N eto FLJ. Fatores sócio-econômicos e ocupacionais e a prática de atividade física regular: estudo a partir de policiais militares em Bauru, São Paulo. M otriz 1998; 4(2):345-350.

20. Candeias NM F. Conceitos de educação e de promoção em saúde: mudanças individuais e mudanças organizacionais. Rev. Saúde Pública 1997; 31(2):209-213.

21. Steven JMD. A Perspective on Educating Physicians for Prevention. Public Health Reports 1982; 97 (3):789-798.

22. Takayanagui AMM M Guimarães FPM . Orientações recebidas do serviço de saúde por pacientes para o tratamento do portador de diabetes mellitus tipo 2 . Rev. Nutr. 2002; 15(1):377-385.

23. Golin CE, Dimatteo MR, Gelber GL. The Role of Patient Participation in the Doctor Visit. Implications for adherence to diabetes care. Diabetes Care 1996; 9(10):1153-1164.

24. Paúl C, Fonseca AM. Psicossociologia da Saúde. Public Health Reports 2001; 12(4):78-90.

25. Chan $Y M$, M olassiotis $A$. The relationship between diabetes knowledge and compliance among Chinese with non-insulin dependent diabetes mellitus in Hong Kong. Journal of Advanced Nursing 1999; 30(2):431-438.

26. Pace EA, Nunes DP, Vigo OK. O conhecimento dos familiares acerca da problemática do portador de diabetes mellitus. Rev. Latino-Americana de Enfermagem 2003; 11(2):312-319.

27. Seyffarth S, Lima PL, Leite CM. Abordagem nutricional em diabetes mellitus. Arq Bras Endocrinol M etabol 1999; 8(2):155-160.

28. Silva AC, Lima CW. Efeito benéfico do exercício físico no controle metabólico do diabetes mellitus tipo 2 a curto prazo. Arq Bras Endocrinol Metabol 2002; 46(5):550-556.

$\overline{\text { Aprovado em 08/01/2007 }}$

Versão final apresentada em13/12/2007 